\title{
Image Contrast Enhancement Techniques: A Comparative Study of Performance
}

\author{
Ismail A. Humied \\ Faculty of Police, \\ Police Academy, Ministry of Interior, Sana'a, \\ Yemen
}

\author{
Fatma E.Z. Abou-Chadi \\ Faculty of Engineering, \\ The British University in Egypt
}

\begin{abstract}
In this paper the performance of four techniques for contrast enhancement of digital images was investigated. The techniques are: histogram equalization (HE), thresholded histogram equalization (WTHE), the low-complexity histogram modification algorithm (LCHM) and a newly developed technique which is a combination of two techniques (HEFGLG): the histogram equalization (HE) and the Fast Gray Level Grouping (FGLG). The performance was compared using different images (gray scale as well as colored) in order to identify which algorithm has the best performance across a variety of images from different sensors and having varying characteristics. Based on the visual quality and the quantitative measures: Absolute Mean Brightness Error (AMBE), the discrete entropy $(\mathrm{H})$, and the measure of enhancement (EME). The experimental results showed that the HEFGLG algorithm outperforms other algorithms. It has the advantage that it has low time complexity since it is a combination of two techniques HE and FGLG, each has low time complexity.
\end{abstract}

\section{Keywords}

Histogram Equalization, Low-complexity histogram modification, Weighted-thresholded histogram equalization, Combined algorithm

\section{INTRODUCTION}

Image contrast enhancement plays a significant role in the field of digital image processing in computer vision applications. It is mainly used to enhance the visual quality of information contained in an image and makes it easier for visual interpretation, understanding as well as image features extraction and analysis by computer vision system [1]. Contrast enhancement is achieved in general, through the histogram equalization i.e. redistribution of intensity values of an input image. The most popular method for contrast enhancement is Histogram equalization (HE) [2]. The basic idea lies on mapping the intensity levels based on the probability distribution of the input intensity levels. It flattens and stretches the dynamics range of the image's histogram and resulting in overall contrast improvement [3]. However, in some applications, the HE algorithm results in an excessively enhanced output image (e.g., display-processing). Moreover, additional limitations which arise out of employing the conventional contrast enhancement techniques include the washed out effect, amplification of background noise, subjective manual manipulation, non-preservation of brightness and the inability to discern localized intensity changes if the images are originally of low contrast - like those pertaining to satellite aerial images and medical images of organs and tissues [4, 5].

Various methods have been proposed for improving the level of contrast enhancement, most of which are obtained through modifications of HE. Wang and Ward [6] suggested modifying the image histogram by weighting and thresholding before histogram equalization (WTHE). The weighting and thresholding is performed by clamping the original histogram at an upper threshold and at a lower threshold, and transforming all the values between the upper and lower thresholds using a normalized power law function.

A histogram modification framework has been suggested in [7] The proposed algorithm is called the low-complexity histogram modification technique (LCHM). It does not require any division operation. It deals with histogram spikes, performs black and white stretching, and adjusts the level of enhancement adaptively so that the dynamic range is better utilized while handling the noise visibility and the natural look requirements.

Recently, Humeid et al. [8] proposed a new algorithm (HEFGLG) which is a combination of Histogram Equalization (HE) [2] and the Fast Gray-Level Grouping (FGLG) [9]. The basic procedure of this method is to segment the original histogram of a low contrast image into two sub-histograms according to the location of the highest amplitude of the histogram components, equalize the left segment of the histogram components using (HE) technique and apply the (FGLG) technique to the right segment of the histogram components. The results have shown that the proposed technique produces better results than the performance of each individual contrast enhancement technique. Moreover, it has the advantage that it is a fully automated technique and it can be applied to a broad variety of images that satisfy the properties mentioned above and suffer from low contrast.

The present work aims to compare the performance of four selected algorithms: HE, WTHE, LCHM and HEFGLG. The main focus is to explore the efficacy of these algorithms for contrast enhancement on a variety of images (gray scale as well as colored) and to identify the algorithm that can be used for automatic contrast enhancement of images obtained from a wide variety of sensors having different characteristics. The criteria adopted to compare performance are subjective and objective. They are based on the visual quality and the calculation of quantitative measures: Absolute Mean Brightness Error (AMBE), the discrete entropy $(\mathrm{H})$, and the measure of enhancement (EME).

In the next section, histogram equalization (HE) is described. Sec. 3 explains the weighed-thresholded contrast enhancement scheme (WTHE). The low-complexity histogram modification (LCHM) algorithm and the combined algorithm (HEFGLG) are presented in Sections 4 and 5, respectively. Section 6 is devoted to results and discussions. Finally, the conclusion is provided in Section 7.

\section{HISTOGRAM EQUALIZATION (HE)}

Histogram is defined as the statistical probability distribution of each gray level in a digital image. Histogram Equalization (HE) [2] is a very popular technique for contrast enhancement of images. Contrast of images is determined by its dynamic range, which is defined as the ratio between the brightest and the darkest pixel intensities. The histogram provides information for the contrast and overall intensity distribution of an image. Suppose an input image I composed of discrete gray levels in the dynamic range $[0, \mathrm{~L}-1]$ then 
the transformation function $\mathrm{C}\left(r_{k}\right)$ is defined as

$$
C\left(r_{k}\right)=\sum_{i=0}^{k} p\left(r_{i}\right)=\sum_{i=0}^{k} \frac{n_{i}}{n}
$$

where $0 \leq C\left(r_{k}\right) \leq 1$ and $k=0,1,2, \ldots, \mathrm{L}-1$. In Equation (1), $n_{i}$ represents the number of pixels having gray level $r_{i}, n$ is the total number of pixels in the input image, and $\mathrm{P}\left(r_{i}\right)$ represents the Probability Density Function (PDF) of the input gray level $r_{i}$. Based on the PDF, the Cumulative Density Function (CDF) is defined as $\mathrm{C}\left(r_{k}\right)$. This mapping in (1) is called Histogram Equalization (HE). Here $C\left(r_{k}\right)$ can easily be mapped to the dynamic range of $[0, \mathrm{~L}-1]$ multiplying it by (L-1).

\section{THE WTHE ALGORITHM}

The histogram of an image with intensity levels in the range $[0, L-1]$ is a discrete function $h(r(k))=n(k)$, where $r(k)$ is the $k$ th intensity level and $n_{k}$ is the number of pixels in the image with intensity $r(k)$. It is common practice to normalize a histogram by dividing each of its components by the total number of pixels in the image, denoted by product $M N$, where, as usual, $M$ and $N$ are the row and column dimensions of the image. Thus, a normalized histogram is given by $P(r(k))=$ $n(k) / M N$, for $\mathrm{k}=0,1,2, \ldots, L-1 . P(r(k))$ is an estimate of the probability of occurrence of intensity level $r(k)$ in an image. Suppose that an input image $I$ with intensity levels in the range $[0, L-1]$ and its histogram was calculated. The weighted-thresholded histogram equalization (WTHE) enhancement method performs histogram equalization (HE) based on a modified histogram [6]. To modify the histogram each original probability density function (PDF) value $P(r(k))$ is replaced by a weighted and thresholded PDF value of $P_{w t}(r(k)) . P_{w t}(r(k))$ is obtained by applying a transformation function $T\left(P_{w t}(r(k))\right)$ to $P(r(k))$, such that

$$
p_{w t}(r(k))=T(P(r(k)))= \begin{cases}P_{u} & \text { if } P(r(k))>P_{u} \\ \left(\frac{P(r(k))-P_{l}}{P_{u}-P_{l}}\right)^{\gamma} \times P_{u} & \text { if } P_{l} \leq P(r(k)) \leq P_{u} \\ 0 & \text { if } P(r(k))<P_{l}\end{cases}
$$

for $k=0,1,2, \ldots, L-1$, The transformation function $T\left(P_{w t}(r(k))\right)$ clamps the original PDF at an upper threshold $P_{u}$ and at a lower threshold $P_{b}$, and transforms all values between the upper and lower thresholds using a normalized power law function with index $\gamma>0$.

After the weighted-thresholded PDF is obtained from Equation (2), the HE transformation function can be applied on the modified histogram [3] using:

$$
\begin{aligned}
& T_{H E}(r(k))=(L-1) \sum_{j=0}^{k} p_{w t}(r(j)) \\
& \text { 4. THE LOW-COMPLEXITY } \\
& \text { HISTOGRAM MODIFICATION } \\
& \text { ALGORITHM (LCHM) }
\end{aligned}
$$

\section{THE LOW-COMPLEXITY HISTOGRAM MODIFICATION ALGORITHM (LCHM)}

In this section, the algorithm of the low-complexity histogram modification (LCHM) [7] is described.

\subsection{Histogram Computation}

In this algorithm, the histogram should be first modified in such a way that the modified histogram, $\hat{h}$, represents the conditional probability of a pixel, given that it has a contrast with its neighbors (denoted by $C$ ). That is, $\hat{h}[i]=P[i \mid C]$, where $P[i \mid C]$ denotes the probability of a pixel having gray level given the event $C$. Then perform histogram equalization on $\hat{h}$ rather than $h$ (original histogram). However, $P[i \mid C]$ can be obtained by counting only those pixels that have contrast, so the modified histogram have not large values of bin. To obtain the histogram, the local variation of each pixel can be used to decide if a pixel has sufficient contrast with its neighbors.

\subsection{Adjusting the level of enhancement}

The Black and White (B\&W) stretching method is applied to the modified histogram, $\hat{h}$ as described in the previous section. The gray level range for $\mathbf{B} \& \mathbf{W}$ stretching is $[0, b]$ and $[w, 255]$, respectively. The modified histogram is the input histogram, $h_{i}$, to the $B \& W$ stretching then the modified histogram becomes

$$
\tilde{h}[n]= \begin{cases}k^{*} h_{i}[n]+\left(1-k^{*}\right) u & b<n<w \\ 1 /(1+\alpha)\left[k^{*} h_{i}[n]+\left(1-k^{*}\right) u\right] & \text { otherwise }\end{cases}
$$

where $n$ and $\quad K^{*}$ are the intensity level and the contribution of the input histogram, respectively, and $\alpha$ is a parameter varies over $[0, \infty]$. To ensure that $h_{i}$ and $u$ have the same normalization, $u$ is obtained using the number of pixels that are included in this histogram.

\section{THE COMBINED TECHNIQUE (HEFGLG)}

The combined technique [8] is composed of the following steps:

\subsection{1 Histogram segmentation}

The position of the highest amplitude histogram component, $P_{\text {hist }}$, can be found on the gray scale. If $\mathrm{P}_{\text {hist }}$ lies inside the left segment of the non-zero histogram components NZHC but not in the first component of the NZHC, the histogram can be segmented into two sub-histograms, the first starting from 0 to $\left.\begin{array}{ll}P_{\text {hist }} & -1\end{array}\right)$ intensity and the second starting from $P_{\text {hist }}$ to maximum intensity level $(L-1)$. If $P_{\text {hist }}$ lies inside the left segment then equalize the left segment of the histogram components using (HE) technique. On the other hand, if $P_{\text {hist }}$ lies inside the right segment of the NZHC or in the first component of the NZHC then FGLG [9] can be used to enhance low contrast image.

\subsection{Piecewise Transformed Function}

Having performed the histogram segmentation according to the position of the highest amplitude histogram component, $P_{\text {hist }}$. the $\mathrm{HE}$ can be applied to first sub-histogram from 0 to $\left(P_{\text {hist }}-1\right)$ and FGLG can be applied to second sub-histogram from $P_{\text {hist }}$ to $L-$ 1. The transformation function using $\mathrm{HE}, T_{H E}(r(k))$, can be expressed as in Equation (2). The transformation function using FGLG is $T_{F G L G}\left(r_{k}\right)$, for $k=P_{\text {hist }}, P_{\text {hist }}+1, P_{\text {hist }}+2, \ldots, L-1$. Therefore the piecewise transformed function $T\left(r_{k}\right)$ can be expressed as follows:

$T(r(k))=T_{H E}\left(r_{(k)}\right)+T_{F G L G}\left(r_{(k)}\right)$

for $k=0,1,2, \ldots, L-1$. Finally, the piecewise transformed function is applied to the original image to reconstruct the optimal enhanced image.

\section{RESULTS AND DISCUSSION}

Although it is desirable to have an objective assessment approach to compare contrast enhancement techniques, unfortunately there is no objective criterion in the literature that gives meaningful results for all image types. There exist some metrics in the literature that measure the contrast in the image based on 
entropy or other measures. If these metrics are used, HE can achieve the best performance even though it may not produce the visually pleasing image, and possibly may produce an unrealistic look. Therefore, it is usually desired to have some quantitative measures in addition to subjective assessment. Hence, the following quantitative measures have been adopted: Absolute Mean Brightness Error (AMBE), the discrete entropy $(H)$, and the measure of enhancement (EME) [10-13].

A time complexity analysis of HE, WTHE, LCHM and HEFGLG was carried out. All techniques were successfully tested on a variety of test images. Only, few figures of the resulted images are shown in this paper.

\subsection{Subjective Assessment}

1) Gray-Scale Images: Figs. 1(a-e) and 3(a-e) show the original test images and their corresponding contrast enhanced versions using the four contrast enhancement algorithms HE, WTHE, LCHM and HEFGLG. The corresponding mapping functions are shown in Figs. 2 and 4, respectively. Comparing the performance of HE, WTHE, LCHM and HEFGLG, it can be noted that HEFGLG gives the best visual quality on the majority of the tested images. However, this is not true with other algorithms. IN general, histogram equalization results in the best utilization of the dynamic range of the pixel values for maximum contrast. However, this often does not mean that the resulting image is better in terms of visual quality. This situation is observed with HE images in Figs. 1(b), and 2(b). In Fig. 1(b) which is the histogram equalized (HE) image of the original image of Fig.1(a). It is clear that the image contrast has been increased at the expense of the amplified noise, and image artifacts. The resulting artifacts lie mostly in the darker regions, which are changing suddenly to the very bright regions. In Fig.1(c), it is clear that WTHE has reduced the effect of HE. However, the resulting image still has some flavor of HE: the text, and the background are still not having the natural-look of the image and the mapping function has a very steep curve.

On the other hand, LCHM and HEFGLG algorithms offer a controllability of the contrast enhancement. LCHM results in images that are visually pleasing than WTHE. Although WTHE thresholds high and low bin values to prevent its undesired effect, it does not produce pleasing results. Since the histogram of LCHM is formed from the conditional probability, it does not have histogram spikes resulting from uniform regions; hence, the LCHM does not produce artifacts as WTHE. The HEFGLG algorithm offers better contrast enhancement than WTHE and LCHM. When the spikes in the original histogram are very large, LCHM does not improve significantly the natural-look of the image comparable to HEFGLG algorithm. Moreover, it should be noted that both WTHE and LCHM utilize larger number of parameters in the transformation function than the HEFGLG algorithm.

Investigation of the mapping function of LCHM shows that it has a smoother curve than that of HE and WTHE. Hence, the contrast enhanced image obtained by the LCHM method is visually more pleasing than HE and WTHE. However the LCHM mapping still has a steep curve resulting in a stretching of a very narrow region into a wider region; range of $[0,27]$ is getting mapped to $[0,175]$ in WTHE, $[0,140]$ in lowcomplexity histogram modification algorithm and range of $[28$, $255]$ is getting mapped to [176, 255], [141 255], respectively. The mapping functions for HE, WTHE and LCHM are showing dark pixels values mapped into very bright pixel values. This is caused by the amplitude histogram components in this type of images which are very high at some location, spikes, on the gray scale and very small in the rest of the gray scale. The mapping function in HEFGLG algorithm is less steeping from the other techniques, so the resulting image is more natural-look. Therefore, the resulting images of Fig. 1(b-d) are not as visually pleasing as Fig. 1(e).

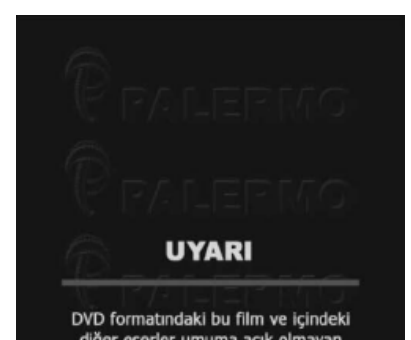

(a)

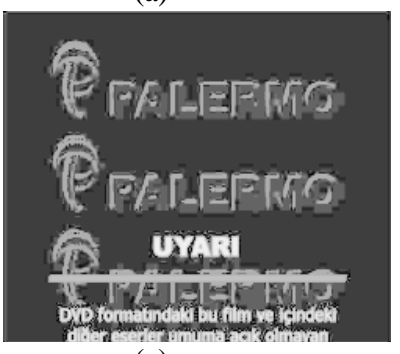

(c)

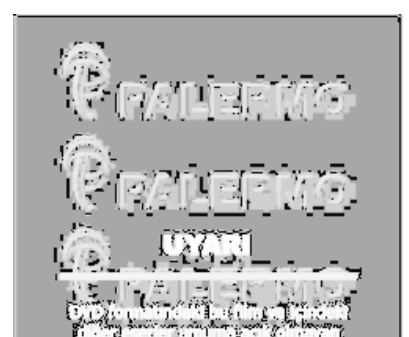

(b)

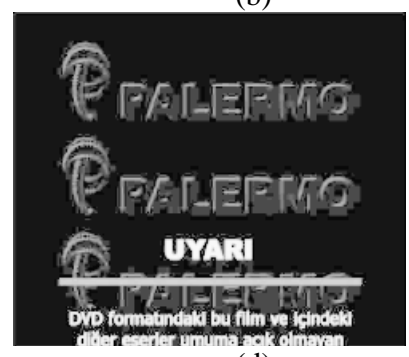

(d)

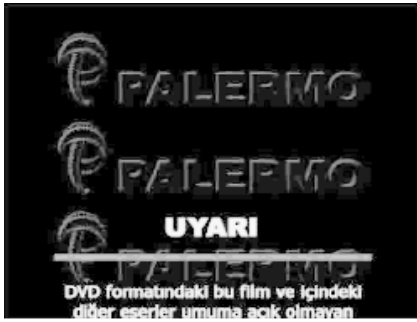

(e)

Fig.1. Results for image Palermo. (a) Original image (b)

enhanced image using $\mathrm{HE}$, (c) enhanced image using WTHE.(d) enhanced image using LCHM and (e) enhanced image using HEFGLG

Fig. 3(b) is the HE image of 3(a). The HE image, looks unnatural and especially, the dominance of the background region results in a big slope in the mapping function around the pixel value of 90 , which results in mapping of range $[60,100]$ into $[120,225]$ as seen in Fig. 4.

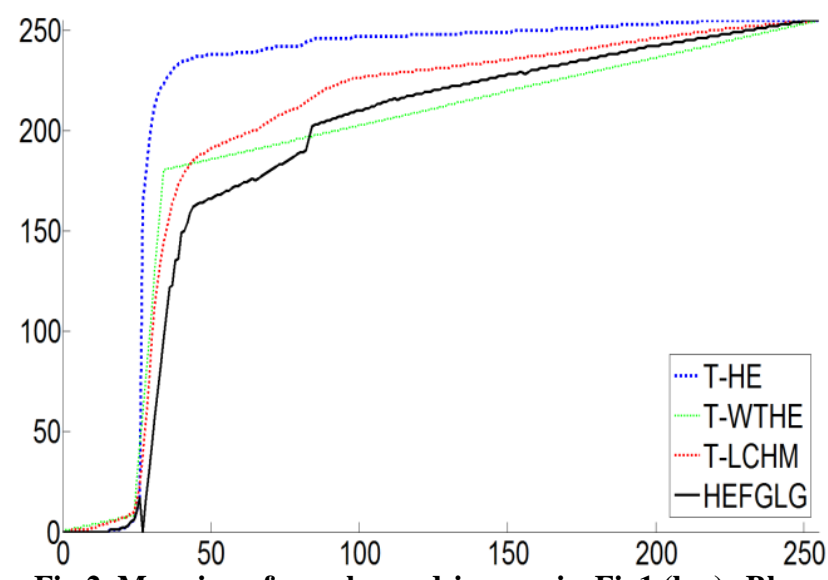

Fig.2. Mappings for enhanced images in Fig1 (b-e). Blue dash-dotted line indicates the $\mathrm{HE}$, green dash-dotted line indicates the WTHE mapping, red dashed for LCHM mapping, and solid line for HEFGLG 


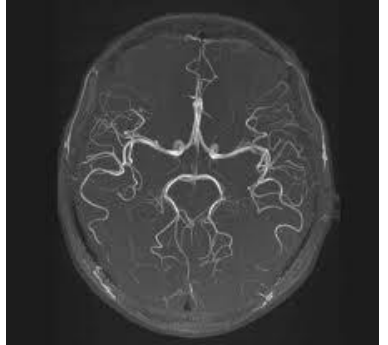

(a)

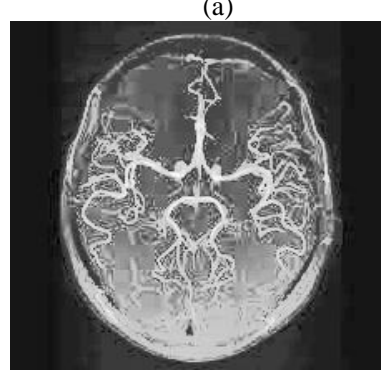

(c)

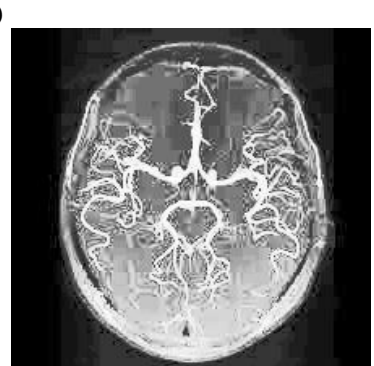

(e)

Fig.3. Results for low contrast image Magnetic Resonance (MR) - Angiography. (a) Original image (b) enhanced image using HE, (c) enhanced image using WTHE. (d) enhanced image using LCHM and (e ) enhanced image using HEFGLG

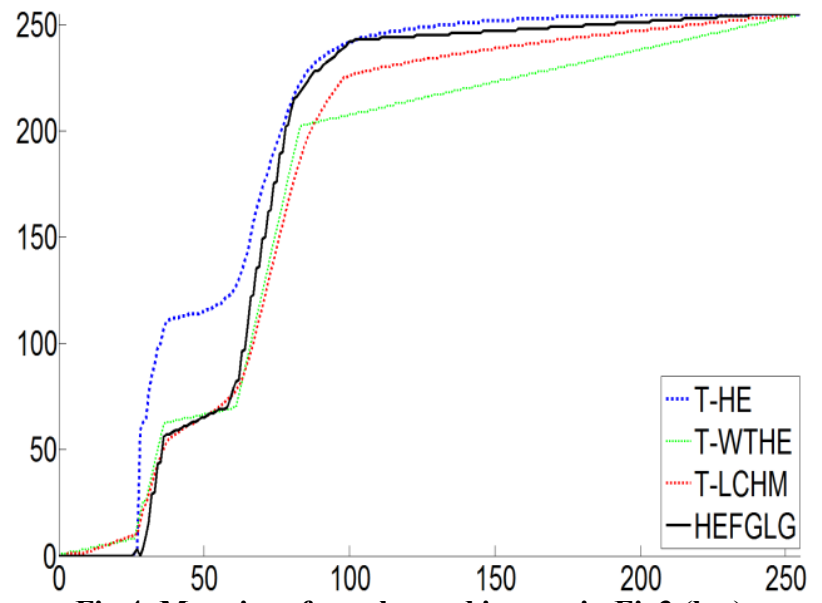

Fig.4. Mappings for enhanced images in Fig3 (b-e)

Unnatural look of the histogram equalized image is lessened using WTHE and LCHM. However, it is not alleviated completely, as shown in Figs. 2(c) and 2(d). This is due to the amplitudes histogram components in this type of images are, again, very high at some location, spikes, on the gray scale and very small in the rest of the gray scale. Large uniform regions in an image cause corresponding bins in the histogram to be very high compared to other bins. However, this has been overcome by the HEFGLG. It avoids the very high bin values and produces a good visual quality result. Note that the contrast of the top object has been much improved and the mapping is more smooth as in Fig. 3(e).

2) Colored Images: Contrast enhancement can be easily applied to colored images. The most obvious way to extend the gray-scale contrast enhancement to colored images is to apply the method to luminance component only and to preserve the chrominance components. One can also multiply the chrominance values with the ratio of their input and output luminance values to preserve the hue [14].

An example using colored images is given in Fig. 5. Fig. 5(b) shows the HE image of Fig.5(a). As it can be seen the image has nonuniform illumination. This becomes more apparent with $\mathrm{HE}$ as it stretches the histogram to increase the contrast. Moreover, the mapping function is not severe as shown in Fig .6. WTHE image (Fig. 5(c)) is visually similar to the original image. The mapping function of this algorithm is linear as clear in Fig. 6. Although the effect of LCHM and the HEFGLG are not severe as HE, it also results in similar artifacts. Therefore, the HE is not the worst in all cases and it can be used to enhance some images, because it is an automatic technique and requires very simple computations, as will be shown in the next section.

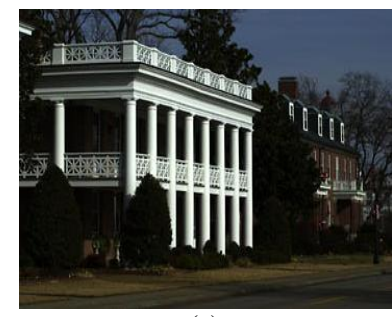

(a)

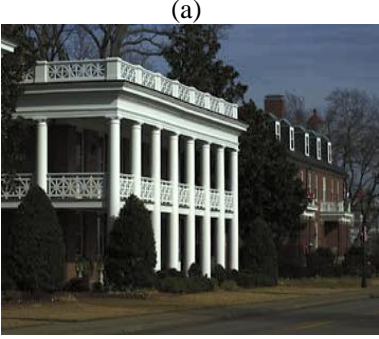

(c)

(e)

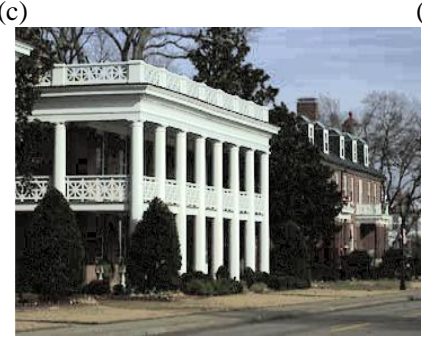

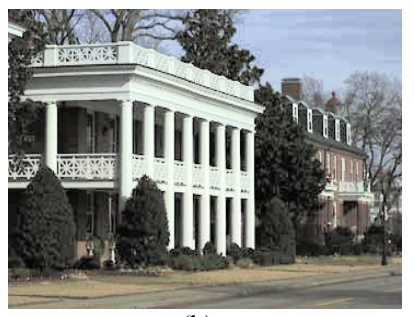

(b)

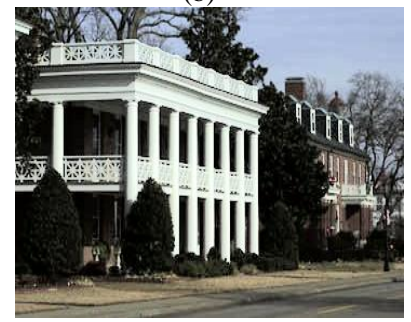

(d)
Fig.5. Results for low contrast image. (a) Original image, (b) enhanced image obtained using HE, (c) enhanced image obtained using WTHE, (d) enhanced image obtained using the LCHM. (e) enhanced image obtained using HEFGLG 


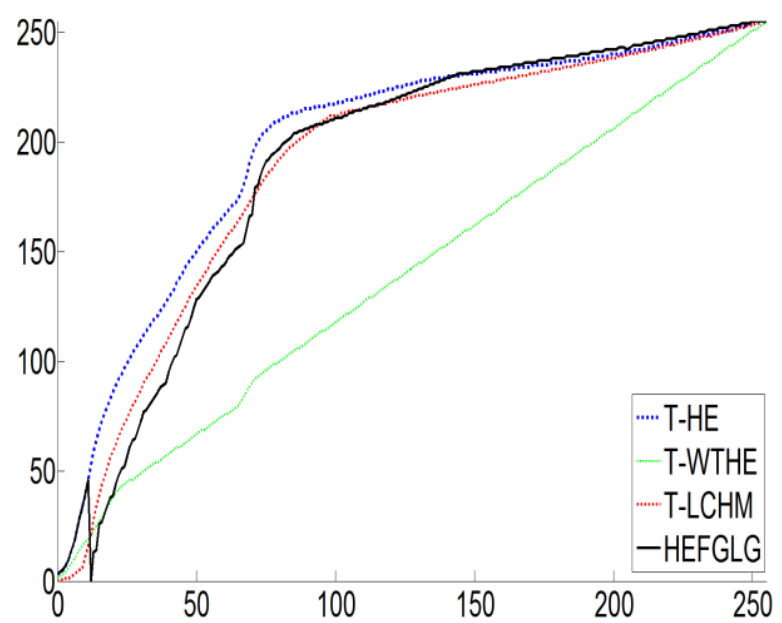

Fig.6. Mappings for enhanced images in Fig5 (a-e)

\subsection{Objective Assessment}

To compare the performance objectively, a number of quantitative measures were calculated. These are: Absolute Mean Brightness Error (AMBE), the discrete entropy $(H)$, and the measure of enhancement (EME) .

AMBE is the absolute difference between original and enhanced image. It is defined as

$$
\mathrm{AMBE}=|E(x)-E(y)|
$$

where $E(x)$ is the average intensity of input image and $E(y)$ is the average intensity of enhanced image.

The discrete entropy $(H)$ is used to measure the in formation contents of an image

$$
H=-\sum_{k=0}^{L-1} P(k) \log P(k)
$$

where $P(k)$ is the probability density function of the $k$ th gray level. A higher value of $H$ indicates an image with richer details.

The measure of enhancement (EME) approximates an average contrast in the image by dividing image into nonoverlapping blocks, finding a measure based on minimum and maximum intensity values in each block, and averaging them.

Table 1 illustrates the calculated values of the above parameters. Comparison of AMBE values shows that HEFGLG outperforms HE, WTHE and LCHM in all images except the colored image. Although HE gives a larger AMBE value than other algorithms in the colored image, it does not necessarily mean they are less faithful to the original image. Preserving the mean brightness does not always mean preserving the natural look of an image. Visual comparison, on the other hand, shows that the visually closest equalized image to the original color image is obtained using HE.

Comparing the values of $H$ shows that the performance of HEFGLG is similar to LCHM and WTHE and all of them outperform $\mathrm{HE}$ in all images except the colored image. Normally, one would expect HE to give higher discrete entropy value as $\mathrm{HE}$ results in more uniform histogram distribution. However, HE results in bin grouping and this decreases the $\mathrm{H}$ value.

Comparison of EME values shows that HEFGLG outperforms other techniques in all images except the first image of Fig.1. Since EME measures a form of contrast, it is not surprising that HE gives the highest value in Fig.1 even though it does not produce the most visually pleasing image.

\subsection{Complexity Comparison}

The time complexity of HE, WTHE, LCMH and HEFGLG was compared for an $\mathrm{M} \times \mathrm{N}$ image (Table 2). It represents the total time of obtaining the contrast enhanced image for each technique.

For HE, computing the histogram requires $\mathrm{O}(\mathrm{MN})$ time. Calculating the mapping function from the histogram requires $\mathrm{O}\left(\mathrm{L}^{\mathrm{B}}\right)$ time, where $\mathrm{L}$ is the total number of gray levels on the grayscale and $\mathrm{B}$ is the number of bits used to represent the pixel values. Moreover, obtaining the enhanced image using the mapping function requires $\mathrm{O}(\mathrm{MN})$ time. Hence, the total time complexity of $\mathrm{HE}$ is $\mathrm{O}\left(2 \mathrm{MN}+\mathrm{L}^{\mathrm{B}}\right)$ [7].

Table 1: The AMBE, $H$ and EME values of the images in Figs.1, 3 and 5.

\begin{tabular}{|c|c|c|c|c|c|}
\hline Parameter & Technique & $\begin{array}{c}\text { Image } \\
1\end{array}$ & $\begin{array}{c}\text { Image } \\
2\end{array}$ & $\begin{array}{c}\text { Image } \\
3\end{array}$ & Average \\
\hline \multirow{4}{*}{ AMBE } & HE & 0.58 & 0.27 & 0.25 & 0.37 \\
\cline { 2 - 6 } & WTHE & 0.23 & 0.13 & 0.05 & 0.14 \\
\cline { 2 - 6 } & LCHM & 0.10 & 0.13 & 0.18 & 0.14 \\
\cline { 2 - 6 } & HEFGLG & 0.004 & 0.12 & 0.19 & 0.10 \\
\hline \multirow{5}{*}{ H } & Original & 2.89 & 5.49 & 7.14 & 5.17 \\
\cline { 2 - 6 } & HE & 2.68 & 5.15 & 7.77 & 5.20 \\
\cline { 2 - 6 } & WTHE & 2.71 & 5.18 & 7.33 & 5.07 \\
\cline { 2 - 6 } & LCHM & 2.81 & 5.35 & 7.68 & 5.28 \\
\cline { 2 - 6 } & HEFGLG & 2.80 & 5.20 & 7.67 & 5.22 \\
\hline \multirow{5}{*}{ EME } & Original & 7.80 & 8.77 & 21.08 & 12.55 \\
\cline { 2 - 6 } & HE & 27.67 & 20.71 & 29.83 & 26.07 \\
\cline { 2 - 6 } & WTHE & 21.43 & 23.08 & 21.81 & 22.11 \\
\cline { 2 - 6 } & LCHM & 23.58 & 23.66 & 31.22 & 26.15 \\
\cline { 2 - 6 } & HEFGLG & 21.05 & 26.55 & 31.47 & 26.36 \\
\hline
\end{tabular}

The WTHE algorithm requires $\mathrm{O}(\mathrm{MN})$ time for calculating the histogram. The modified histogram for each bin requires $\mathrm{O}\left(\mathrm{L}^{\mathrm{B}}\right)$ time, the computation of the mapping function requires $\mathrm{O}\left(\mathrm{L}^{\mathrm{B}}\right)$ time, and obtaining the enhanced image using the mapping function requires $\mathrm{O}(\mathrm{MN})$ time. Hence, the total time complexity of $\mathrm{LCHM}$ is $\mathrm{O}\left(2 \mathrm{MN}+2 \mathrm{~L}^{\mathrm{B}+1}\right)[7]$.

The LCHM algorithm, computing the histogram requires $\mathrm{O}(\mathrm{MN})$ time, the modified histogram for each bin requires $\mathrm{O}\left(\mathrm{L}^{\mathrm{B}}\right)$ time, the mapping function requires $\mathrm{O}\left(\mathrm{L}^{\mathrm{B}}\right)$ time, and that of obtaining the enhanced image using the mapping function requires $\mathrm{O}(\mathrm{MN})$ time. Hence, the total time complexity of the $\mathrm{LCHM}$ is $\mathrm{O}\left(2 \mathrm{MN}+2 \mathrm{~L}^{\mathrm{B}+1}\right)[7]$.

As for HEFGLG algorithm, the computational complexity for calculating gray-level transformation functions in the optimal GLG process is $\mathrm{O}\left(\mathrm{L}^{2 \mathrm{~B}}\right)$ [9]. However, since the gray-level transformation function is calculated only once in the fast GLG process, the computational complexity for multiplication operations is $\mathrm{O}\left(\mathrm{L}^{\mathrm{B}}\right)$. Computation of the histogram and obtaining the enhanced image using the mapping function requires $\mathrm{O}(2 \mathrm{MN})$ time. Hence, the total time complexity of FGLG is $\mathrm{O}\left(2 \mathrm{MN}+\mathrm{L}^{\mathrm{B}}\right)$. Since the HE time complexity is $\mathrm{O}\left(2 \mathrm{MN}+\mathrm{L}^{\mathrm{B}}\right)$ and finding the position of the highest amplitude histogram component, Phist, on the gray scale is $\mathrm{O}\left(\mathrm{L}^{\mathrm{B}}\right)$, hence, 
the total time complexity of combined algorithm is $\mathrm{O}(2 \mathrm{MN}+$ $\left.3 \mathrm{~L}^{\mathrm{B}}\right)$.

As a result, WTHE and LCHM algorithms have the same time complexity. This is slightly worse than HE as HE does not require the modification of the histogram before equalization. The computational complexity of the HEFGLG algorithm is less than those of WTHE and LCHM.

\section{Table 2: General comparison between the six techniques}

\begin{tabular}{|c|c|c|c|}
\hline Algorithm & General & Automatic & $\begin{array}{c}\text { Computational } \\
\text { Complexity }\end{array}$ \\
\hline HE & No & $\sqrt{ }$ & $O(2 M N+L)$ \\
\hline WTHE & Yes & $\mathrm{X}$ & $O(2 M N+2 L)$ \\
\hline FGLG & Yes & $\mathrm{X}$ & $O(2 M N+2 L)$ \\
\hline HEFGLG & Yes & $\sqrt{ }$ & $O(2 M N+L)$ \\
\hline
\end{tabular}

\section{CONCLUSION}

In this paper, a comparative study of performance of four contrast enhancement techniques has been presented. These are: the histogram equalization (HE), thresholded histogram equalization (WTHE), the low-complexity histogram modification algorithm (LCHM) and HEFGLG technique which is a combination of two techniques: the histogram equalization (HE) and the Fast Gray Level Grouping (FGLG). Qualitative and quantitative measures were adopted for the comparison.

HE is a very popular algorithm. It is capable of finding hidden image information in darkness. It has the advantage of being automatic, simple and low complexity, but its final image appearance is often not very good. While WTHE and LCHM produced a higher quality image than HE, but they can not fulfill certain special application purposes, such as in case of the image has high amplitude histogram components at some location, spikes and very small amplitudes in the rest of the gray scale. Moreover, both WTHE and LCHM utilize a large number of parameters in the transformation function and their computational complexity is timely consuming. The experimental results show the effectiveness of the HEFGLG algorithm compared to the other three contrast enhancement algorithms. Resulting images are visually pleasing, artifact free, and natural looking. It has a low time complexity because it is a combination of two techniques HE and FGLG, where each has low complexity. It can be concluded that the combined technique HEFGLG can be implemented for full automation across a broad variety of low-contrast images.

\section{REFERENCES}

[1] Rafael C. Gonzalez and Richard E. Woods, Digital Image Processing ( $3^{\text {rd }}$ Edition), Prentice Hall, August 2008.
[2] Frank Y. Shih J, Image Processing and Pattern Recognition Fundamentals and Techniques, the Institute of Electrical and Electronics Engineers, Inc, Hoboken, New Jersey- Canada, Wiley and Sons, 2010.

[3] R. Hummel, 'Histogram modification techniques', Computer Graphics and Image Processing, vol.4, no.3, pp. 209-224, 1975

[4] Senthilkumaran, N., Thimmiaraja, J.: Histogram equalization for image enhancement using MRI brain images. In: 2014 World Congress on Computing and Communication Technologies (WCCCT), pp. 80-83. IEEE, 2014.

[5] Kaur, M., Kaur, J., Kaur, J.: Survey of contrast enhancement techniques based on histogram equalization. International Journal of Advanced Computer Science and Applications, 2011

[6] Q. Wang and R. K. Ward, "Fast image/video contrast enhancement based on weighted thresholded histogram equalization," IEEE Trans. Consum. Electron., vol. 53, no. 2, pp. 757-764, May 2007.

[7] T. Arici, S. Dikbas and Y. Altunbasak, "A Histogram Modification Framework and Its Application for Image Contrast Enhancement," IEEE Trans. Image. Process., vol. 18, no. 9, pp. 1921-1935, Sept 2009.

[8] Ismail A. Humied, Fatma E.Z. Abou-Chadi and Magdy Z. Rashad, "A new combined technique for automatic contrast enhancement of digital images", Egypt Inform J 2012 .

[9] Z. Chen, B. R. Abidi, D. L. Page, and M. A. Abidi, "Graylevel grouping (GLG): an automatic method for optimized image contrast enhancement-part I: the basic method," IEEE Transactions on Image Processing, vol. 15, no. 8, pp. 2290-2302, Aug. 2006.

[10] G. Ravichandran and V. Magudeeswaran, "An Efficient Method for Contrast Enhancement in Still Images using Histogram Modification Framework, ”Journal of Computer Science, no. 5, P. 775-779, 2012.

[11] Yen-Ching Chang and Chun-Ming Chang, , 'A Simple Histogram Modification Scheme for Contrast Enhancement', IEEE Transactions on Consumer Electronics, Vol. 56, No. 2., 2010.

[12] S.-D. Chen and A. Ramli, "Minimum mean brightness error bi-histogram equalization in contrast enhancement," IEEE Trans. Consum. Electron., vol. 49, no. 4, pp. 1310-1319, Apr. 2003

[13] Saravanan S, and P.Siva Kumar, "Image Contrast Enhancement Using Histogram Equalization Techniques: Review," International Journal of Advances in Computer Science and Technology, vol. 3, no. 3, pp. 163-172, March 2014 